[Article]

www.whxb.pku.edu.cn

\title{
钯镍合金在玻碳电极上电结晶机理
}

\author{
岳俊培＼cjkstart杨防祖* 田中群＼cjkstart周绍民 \\ (厦门大学化学化工学院, 固体表面物理化学国家重点实验室, 福建厦门 361005)
}

\begin{abstract}
摘要: 在钯镍合金电解液中, 采用循环伏安和计时安培实验方法, 运用 Scharifker 和 Hills 模型、Heerman 和 Tarallo 模型揭示钯镍合金的成核机理. 结果表明, 钯镍合金在玻碳电极上发生成核过程. Scharifker 和 Hills 模 型分析表明, 钯镍合金更符合扩散控制下的三维生长的连续成核机理. 借助于 Heerman 和 Tarallo 模型, 拟合得 到钯镍合金的成核和生长的动力学参数. 拟合结果显示, 电位阶跃从 $-0.85 \mathrm{~V}$ 负移至 $-0.92 \mathrm{~V}$ (vs SCE), 钯镍合 金在玻碳电极上的成核速率从 $0.83 \mathrm{~s}^{-1}$ 增加到 $7.71 \mathrm{~s}^{-1}$, 成核密度数从 $2.77 \times 10^{4} \mathrm{~cm}^{-2}$ 提高至 $7.09 \times 10^{4} \mathrm{~cm}^{-2}$.
\end{abstract}

关键词: 钯镍合金; 电结晶; 循环伏安; 计时安培曲线

中图分类号: 0646

\section{Electrocrystallization of Pd-Ni Alloys on Glassy Carbon Electrode}

\author{
YUE Jun-Pei YANG Fang-Zu* TIAN Zhong-Qun ZHOU Shao-Min \\ (State Key Laboratory of Physical Chemistry of Solid Surfaces, College of Chemistry and Chemical Engineering, \\ Xiamen University, Xiamen 361005, Fujian Province, P. R. China)
}

\begin{abstract}
Using the Scharifker and Hills model and Heerman and Tarallo model, cyclic voltammetry and chronoamperometry were used to study the nucleation mechanism upon the electrodeposition of a Pd-Ni alloy from an electrolyte. The results show that the electrodeposition of the Pd-Ni alloy onto glassy carbon electrode consisted of nucleation and growth, and the nucleation process according to the Scharifker and Hills model follows three dimension-progressive nucleation, which is controlled by the diffusion of the electroactive species. By applying the Heerman and Tarallo model, the kinetic parameters associated with the crystal nucleation and growth processes were obtained. The nucleation rate constant and the density of active nucleation sites increased from 0.83 to $7.71 \mathrm{~s}^{-1}$ and from $2.77 \times 10^{4}$ to $7.09 \times 10^{4} \mathrm{~cm}^{-2}$, respectively, as the step potential was changed from -0.85 to $-0.92 \mathrm{~V}$ ( $v s$ SCE).
\end{abstract}

Key Words: Pd-Ni alloy; Electrocrystallization; Cyclic voltammetry; Chronoamperometry

\section{1 引言}

金属电沉积(电结晶)过程的研究不仅能构筑及 解释金属沉积的机理, 而且对实际应用具有广泛的 指导意义. 众所周知, 金属的沉积经历成核和生长 两个主要过程. 在金属沉积过程中, 沉积过电位对 成核和生长过程有较大的影响. 通常, 恒电位阶跃
计时安培曲线是研究金属电结晶机理的常用方法. Fleischmann 及其合作者 ${ }^{1,2}$ 最早应用此方法研究二 氧化铅在铅和铂上的沉积过程, 并提出了基本的成 核方程: ${ }^{3}$

$$
\frac{\mathrm{d} N}{\mathrm{~d} t}=A N_{0} \mathrm{e}^{(-A t)}
$$

式中, $N_{0}$ 代表基底表面饱和活性位数 $\left(\mathrm{cm}^{-2}\right)$, 也即是

Received: December 16, 2010; Revised: February 18, 2011; Published on Web: May 4, 2011.

*Corresponding author. Email: fzyang@xmu.edu.cn; Tel: +86-592-2185957; Fax: +86-592-2181436

The project was supported by the National Natural Science Foundation of China $(20873114,20833005)$ and National Key Basic Research Program of China (2009CB930703).

国家自然科学基金(20873114, 20833005)及国家重点基础研究发展计划(2009CB930703)资助项目

C. Editorial office of Acta Physico-Chimica Sinica 
成核密度数; $A$ 为成核速率常数 $\left(\mathrm{s}^{-1}\right) ; t$ 为时间 (s). 该 方程对金属电结晶过程的研究具有重要的指导意 义. 随后的基于计时安培曲线建立的电结晶成核理 论模型, 大都是建立于该方程的基础之上.

较为重要的成核模型有 Scharifker 和 Hills 模型 (SH)、 ${ }^{4,5}$ Scharifker 和 Mostany 模型 (SM)、 ${ }^{6}$ SluytersRehbach 等研究者的模型 $(\mathrm{SR})^{7}$ 以及 Heerman 和 Tarallo 模型(HT) ${ }^{8}$ 值得注意的是, 这些模型都是建 立在扩散控制、三维生长下的成核模型的基础之上 的. SH 模型被广泛应用于判断成核机理(连续成核、 瞬时成核); $\mathrm{SM}$ 模型对 $\mathrm{SH}$ 模型进行了改善, 被广泛 用来求算成核参数 $N_{0}$ 和 $A$; SR 模型对 $\mathrm{SM}$ 模型进行 了修正, 认为在扩散中心发生重叠前, 扩散模型应 该为平面扩散, 扩散层厚度恒定为 Nernst 扩散层厚 度. 尽管 SM 模型与 SR 模型总体相似, 但根据二者 得出的实验结果却有很大的差别. ${ }^{9}$ Heerman 和 Tarallo 综合了 SR 模型中扩散中心重叠前平面扩散 的厚度为 Nernst 扩散层厚度, 以及 $\mathrm{SM}$ 模型在展开 覆盖时的圆柱扩散, 认为扩散层的厚度不是恒定的 Nernst 扩散层厚度, 而是成核密度数和时间的函 数. ${ }^{8}$ 因此, 目前, 研究者多用 HT 模型进行计时安培 曲线拟合, 从而得到金属成核过程的相关参数.

钯镍合金因其良好的物理和化学性质, 如较好 的耐蚀性, 较低的接触电阻, 较高的硬度以及光亮 的外观, 被广泛应用于电子工业、装饰性行业、微机 电加工行业中. ${ }^{10-12}$ 此外, 钯镍合金还因其卓越的催 化性质而被应用于不饱和烃的加氢反应, ${ }^{13}$ 硝基的 还原反应, ${ }^{14}$ 以及甲醇、乙醇、甲酸等小分子的催化 反应. ${ }^{15-17}$ 尽管钯在不同电极表面成核及生长的研究 有许多报道, ${ }^{18-20}$ 但钯镍合金电结晶过程的理论研究 并不充分, 这严重制约了人们对钯镍合金电结晶机 理的理论认识及其应用的理论指导.

在合金的电结晶过程中, 成核物质不是单一金 属, 成核过程中合金金属之间发生相互作用. 不同 的沉积(过)电位下, 合金沉积的组分含量可能变化, 并导致合金的密度改变; 从而将导致成核机理判断 (实验结果偏离理论曲线)和成核过程参数计算的困 难与误差. 因此, 在电结晶机理研究及成核参数的 计算过程中, 人们基本上采用单金属体系、简单组 成及高度稀释后的电解液, 而非合金体系以及可实 际应用的电解液. 为了更接近于实际体系及得到的 研究结果, 本文以可工业化应用的钯镍合金电解液 为研究体系, 采用循环伏安以及计时安培曲线方
法, 考察钯镍合金在玻碳电极上的电沉积过程, 运 用 SH 模型揭示钯镍合金的成核机理, 应用 HT 模型 拟合求得钯镍合金电结晶过程的成核参数.

\section{2 实 验}

循环伏安曲线和计时安培曲线用 model 660 电 化学工作站(CH Instrument, USA)来得到. 实验采用 三电极体系, 工作电极为玻碳圆盘电极 (GCE) $(d=4$ $\mathrm{mm}$ ), 对电极为铂片电极, 参比电极为饱和甘录电极 (SCE), 文中电位都相对于 SCE. 电解液为 0.020 $\mathrm{mol} \cdot \mathrm{L}^{-1} \mathrm{Pd}\left(\mathrm{NH}_{3}\right)_{2} \mathrm{Cl}_{2}, 0.010 \mathrm{~mol} \cdot \mathrm{L}^{-1} \mathrm{NiSO}_{4} \cdot 6 \mathrm{H}_{2} \mathrm{O}$, $1.0 \mathrm{~mol} \cdot \mathrm{L}^{-1} \mathrm{NH}_{4} \mathrm{Cl}, 0.8 \mathrm{~mol} \cdot \mathrm{L}^{-1} \mathrm{H}_{3} \mathrm{BO}_{3}, \mathrm{pH}$ 为 8.0 (用 氨水调节), 用国药集团化学试剂有限公司生产的分 析纯试剂和超纯水配制. 实验前, 玻碳电极首先在 砂纸上打磨抛光, 用 0.3 和 $0.05 \mu \mathrm{m}$ 三氧化二铝抛光 粉分别抛光, 在丙酮中超声波清洗 2 min 后, 再在超 纯水中超声波清洗 $2 \mathrm{~min}$, 最后用超纯水冲洗干净并 使用.

循环伏安实验在室温下进行 $\left(25^{\circ} \mathrm{C}\right)$, 电位 从 $-100 \mathrm{mV}$ 负向扫描. 计时安培实验中, 阶跃电位 分别为 $-0.85 、-0.87 、-0.88 、-0.90$ 和 $-0.92 \mathrm{~V}$.

\section{3 结果与讨论}

\section{1 钯镍合金电沉积的循环伏安分析}

图 1 显示了玻碳电极在钯镍合金电解液中, 不 同阴极截止电位及不同扫描圈数的循环伏安曲线; 电位从平衡电位负向移动, 高截止电位为 $0.40 \mathrm{~V}$; 曲 线 $1 、 2$ 和 3 的低截止电位分别为 $-0.80 、-1.00$ 和 $-1.20 \mathrm{~V}$, 曲线 4 为低截止电位为 $-1.20 \mathrm{~V}$ 时的第

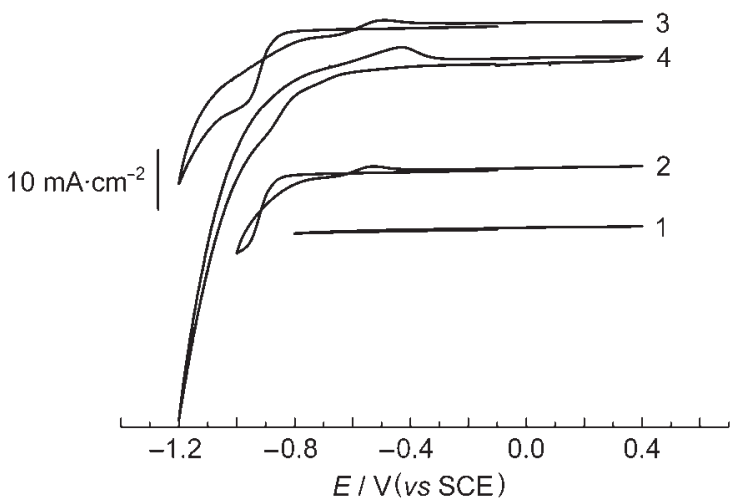

图 1 玻碳电极在钯镍合金电解液中的循环伏安曲线

Fig.1 Cyclic voltammograms of glassy carbon electrode (GCE) in the electrolyte of Pd-Ni alloy

curves $1,2,3$ for the low terminal potentials of $-0.80,-1.00,-1.20 \mathrm{~V}$, respectively, curve 4 for the second scanning circle of curve 3 ; scan rate: $50 \mathrm{mV} \cdot \mathrm{s}^{-1}$ 
二圈扫描, 扫描速率为 $50 \mathrm{mV} \cdot \mathrm{s}^{-1}$. 通过不同截止电 位的循环伏安曲线可以清楚地对比分析各个阴阳 极峰所代表的电极过程. 当截止电位为 $-0.80 \mathrm{~V}$ 时 (曲线 1), 可以明显地发现, 在整个电位区间内没有 明显的电流变化过程, 说明没有发生金属的还原及 氧化过程. 当截止电位为 $-1.00 \mathrm{~V}$ 时 (曲线 2), 在 $-0.82 \mathrm{~V}$ 附近开始出现起波阴极电流, 归因于钯 镍合金的共沉积; 阳极方向扫描时, 在 $-0.53 \mathrm{~V}$ 附近 出现一氧化电流峰, 归因于钯镍合金沉积物的溶 出. 当截止电位为 $-1.20 \mathrm{~V}$ 时 (曲线 3 ), 玻碳电极上的 阴阳极过程与曲线 2 的相似, 不同之处在于, 在负 于 $-1.06 \mathrm{~V}$ 的电位区间出现了析氢反应电流. 值得 注意的是, 曲线 2 和曲线 3 在 $-0.91--0.60 \mathrm{~V}$ 区间 均出现了一个电流环, 说明钯镍合金在玻碳电极上 沉积经历了成核过程. ${ }^{21}$ Fletcher 等 ${ }^{22}$ 认为成环电位 应该和金属沉积的平衡电位一致. 根据图 1 曲线 2 和 3 , 成环电位为 $-0.60 \mathrm{~V}$, 故钯镍合金在玻碳电极上 沉积的平衡电位应为 $-0.60 \mathrm{~V}$. 钯镍合金在玻碳电极 上的起始沉积电位为 $-0.82 \mathrm{~V}$. 平衡电位和沉积电位 的差值为 $220 \mathrm{mV}$, 说明钯镍合金具有较大的成核过 电位, 并清楚出现电流环. 图 1 中曲线 4 表示对应于 曲线 3 终止电位为 $-1.20 \mathrm{~V}$ 时, 玻碳电极在钯镍合金 电解液中的第二圈循环伏安曲线. 从曲线 4 可以清 楚地发现, 经过曲线 3 循环扫描、在覆盖有钯镍合金 沉积物的玻碳电极上, 钯镍合金的沉积电位更正, 为 $-0.53 \mathrm{~V}$ 左右; 析氢电流更大且无电流成环现象. 电流环在循环伏安曲线第二圈消失也能在钯电沉 积过程中发现. ${ }^{23}$ 这一结果表明, 玻碳电极表面上钯

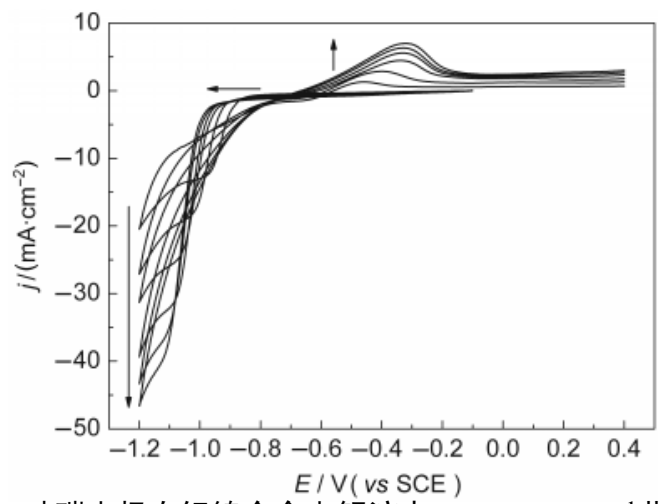

图 2 玻碳电极在钯镍合金电解液中 $50-500 \mathrm{mV} \cdot \mathrm{s}^{-1}$ 范围 扫描速率下的循环伏安曲线

Fig.2 Cyclic voltammograms of glassy carbon electrode (GCE) in the Pd-Ni electrolyte between 50 and $500 \mathrm{mV} \cdot \mathrm{s}^{-1}$ scan rates

The scan rates following the arrow are 50, 100, 200, 300, 400, and $500 \mathrm{mV} \cdot \mathrm{s}^{-1}$ in order.
镍合金沉积物对钯镍合金的进一步电沉积及析氢 反应具有催化作用, 且没有经历成核过程.

为进一步探索钯镍合金的电沉积过程, 给出玻 碳电极在钯镍合金电解液中不同扫描速率的循环 伏安曲线, 结果如图 2 所示. 随着扫描速率的提高, 钯镍合金的沉积峰电位逐渐负移, 氧化峰电位逐渐 正移, 还原峰电流及氧化峰电流均逐渐增大. 显然, 钯镍合金的沉积不是可逆的电极过程. 根据峰电流 与扫描速率平方根之间的 Randles-Sevcik 公式, ${ }^{24,25}$ 可进一步判断该电极过程. 钯镍合金沉积峰电流、 氧化峰电流与扫描速率平方根之间的关系如图 3 所 示. 由图 3 可知, 峰电流与扫描速率的平方根成良好 的线性关系, 说明钯镍合金电沉积在此实验条件下 属于扩散控制下的不可逆过程.

\section{2 钯镍合金电沉积的计时安培分析}

为了解钯镍合金电结晶的动力学过程, 我们得 到了不同阶跃电位 $(-0.85,-0.87,-0.88,-0.90$ 和 $-0.92 \mathrm{~V})$ 下的计时安培曲线, 结果示于图 4. 由图 4 可知, 当电位阶跃到一个适当的过电位时, 经历一 段时间的诱导后, 电流增加达到最大, 后下降, 直至 基本稳定. 不同阶跃电位下的诱导时间不一, 在较
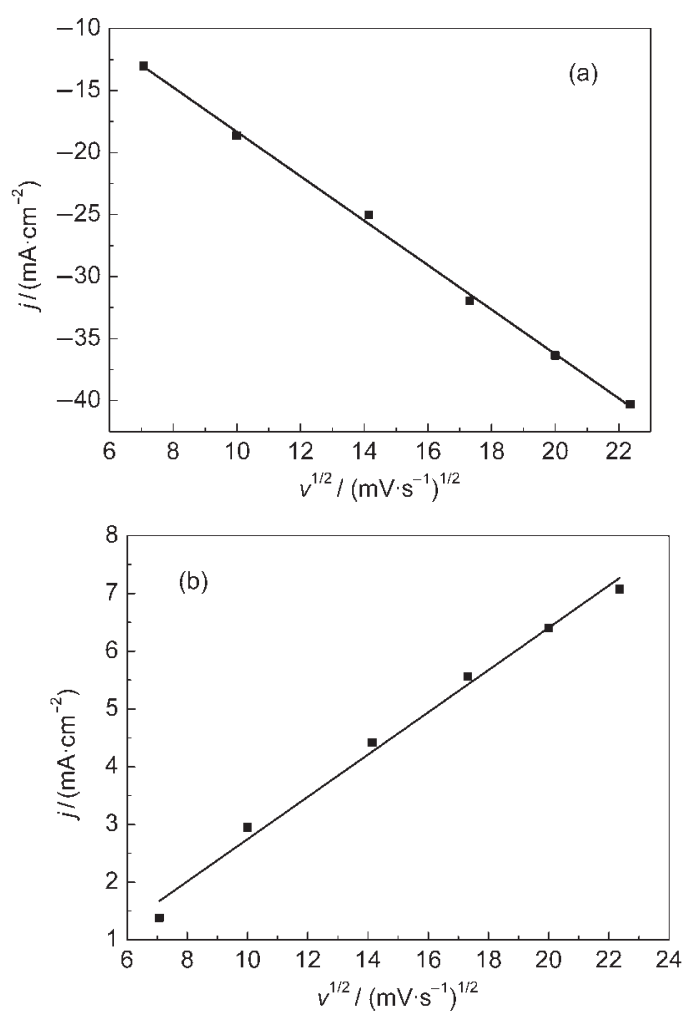

图 3 钯镍合金沉积峰电流(a)和氧化峰电流(b)与 扫描速率的平方根关系曲线.

Fig.3 Variations in current density of cathodic (a) and anodic (b) peaks with square root of scan rate 


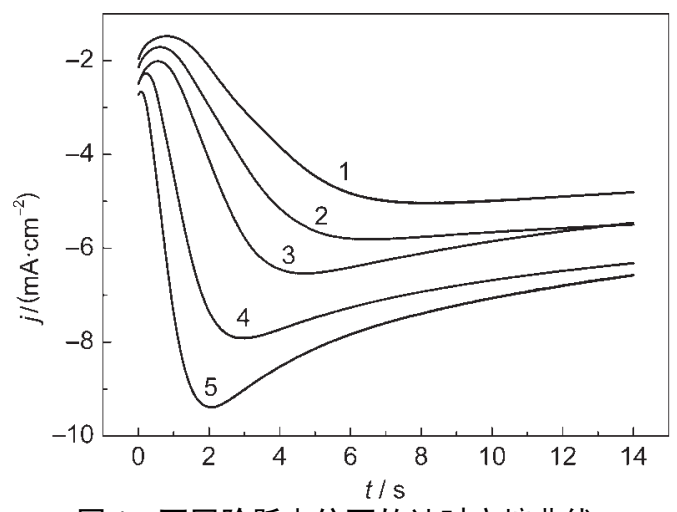

图4 不同阶跃电位下的计时安培曲线

Fig.4 Potentiostatic current transients for the nucleation of Pd-Ni on GCE at different step potentials

curves $1,2,3,4$, and 5 for step potentials of $-0.85,-0.87$, $-0.88,-0.90,-0.92 \mathrm{~V}$, respectively

正的阶跃电位下, 诱导时间较长; 较负的阶跃电位 下, 诱导时间则很短. 同时, 随着阶跃电位的负移, 达到峰电流的时间越来越短. 计时安培曲线呈现了 典型的扩散控制下三维生长的成核过程的曲线特 征. ${ }^{4}$ 根据 Scharifker 和 Hills 模型推导出的恒电位暂 态曲线, $I^{2} / I_{\mathrm{m}}^{2}\left(I\right.$ 为响应电流密度, $I_{\mathrm{m}}$ 为最大响应电流 密度)与 $t / t_{\mathrm{m}}$ ( $t$ 为响应时间, $t_{\mathrm{m}}$ 为最大响应电流密度时 的时间)的比值的无因次关系，

对于瞬时成核: $I^{2} / I_{\mathrm{m}}^{2}=\frac{1.9542}{\left(t / t_{\mathrm{m}}\right)}\left\{1-\exp \left[-1.2564\left(t / t_{\mathrm{m}}\right)\right]\right\}^{2}$ 对于连续成核: $I^{2} / I_{\mathrm{m}}^{2}=\frac{1.2254}{\left(t / t_{\mathrm{m}}\right)}\left\{1-\exp \left[-2.3367\left(t / t_{\mathrm{m}}\right)\right]\right\}^{2}$ 根据 $I^{2} / I_{\mathrm{m}}^{2}$ 与 $t / t_{\mathrm{m}}$ 关系, 可以得到瞬时成核和连续成核 理论曲线, 结果如图 5 所示. 图 5 曲线 1 代表瞬时成核 理论曲线, 曲线 2 代表连续成核理论曲线, 曲线 3,4 , $5,6,7$ 分别代表阶跃电位为 $-0.85 、-0.87 、-0.88$ 、

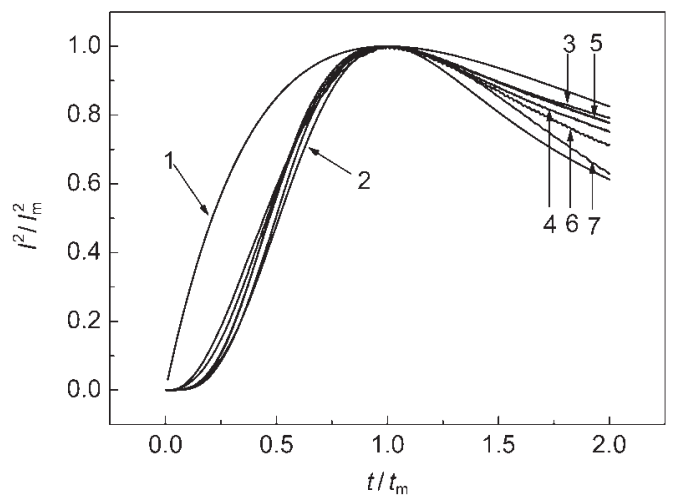

图 5 无因次计时安培曲线对比图

Fig.5 Comparison of theoretical non-dimensional plots instantaneous nucleation curve 1 and progressive nucleation curve 2, to the experimental current transient curves $3,4,5,6$, and 7 with the step potentials of $-0.85,-0.87,-0.88,-0.90$, and $-0.92 \mathrm{~V}$, respectively
$-0.90 、-0.92 \mathrm{~V}$ 的无因次计时安培实验曲线. 可见, 实验曲线更贴近连续成核理论曲线, 说明钯镍合金 在沉积过程中更加符合扩散控制下三维生长连续 成核的成核规律. 值得注意的是, 当 $t / t_{\mathrm{m}}>1$ 时, 实验 曲线逐渐偏离连续成核理论曲线. 显然, 合金的电 结晶过程更加复杂. 造成这一结果的可能原因是析 氢副反应的影响. 循环伏安实验结果说明, 相对于 玻碳电极, 钯镍合金有催化析氢反应的作用. 在钯 镍合金电结晶过程中, 玻碳电极表面覆盖有钯镍物 质, 导致析氢反应的增大. 钯镍合金电结晶初始阶 段 $\left(t / t_{\mathrm{m}} \leq 1\right)$ 成核机理的实验结果与理论模型较为吻 合. SH 模型对合金的电结晶机理研究是否广泛适用 (或需修正), 需要更多的研究证实.

由于 Scharifker 和 Hills 模型得到的时间电流曲 线方程难以求取有效的成核机理参数, ${ }^{9}$ 如成核密度 数, 成核速率常数等. 本文根据 Heerman 和 Tarallo 理论分析求得成核密度数和成核速率常数. Heerman 和 Tarallo 理论关于恒电位下电流密度与时 间关系如下:

$$
\begin{aligned}
& j(t)=z F D c(\pi D t)^{-1 / 2} \frac{\Phi}{\Theta}\left(1-\exp \left[-\alpha N_{0}(\pi D t)^{1 / 2} t^{1 / 2} \Theta\right]\right) \\
& \alpha=2 \pi(2 M D c / \rho)^{1 / 2} ; \Phi=1-\frac{\mathrm{e}^{-A t}}{(A t)^{1 / 2}} \int_{0}^{(A t)^{1 / 2}} \mathrm{e}^{\lambda^{2}} \mathrm{~d} \lambda \\
& \Theta=1-\left(1-\mathrm{e}^{-A t}\right) / A t
\end{aligned}
$$

式中, $j(t)$ 为电流密度 $\left(\mathrm{A} \cdot \mathrm{cm}^{-2}\right), z$ 为转移电子数 $(z=$ $2), F$ 为法拉第常数 $\left(96500 \mathrm{C} \cdot \mathrm{mol}^{-1}\right), D$ 为扩散系数, $c$ 为摩尔浓度, $\rho$ 为金属密度, $A$ 为成核速率常数 $\left(\mathrm{s}^{-1}\right), M$ 为摩尔质量, $N_{0}$ 为表面活性位饱和数 $\left(\mathrm{cm}^{-2}\right)$, 即成核密度数.

Heerman 和 Tarallo 应用基于 Levenberg-Marquardt 运算法则的非线性最小二乘法原理, 以成核 密度数以及成核速率常数为拟合参数, 通过拟合曲 线求得其相应数值. ${ }^{26}$ 本文也应用该法则, 以成核密 度数, 成核速率常数和活性物质的扩散系数为拟合 参数, 借助 Origin 7.5 数据处理软件非线性高级拟合 工具, 拟合求得其数值, 结果示于表 1 , 理论拟合曲 线(点划线)与实验曲线(实线)对比示于图 6. 拟合时 用到的参数值: $z=2, F=96500 \mathrm{C} \cdot \mathrm{mol}^{-1}, c=3 \times 10^{-5}$ $\mathrm{mol} \cdot \mathrm{cm}^{-3}$. 能量色散谱分析可知, 在不同阶跃电位下 钯镍合金的组成基本不变, 镍的质量分数在 $20 \%$ 左 右, 原子分数在 $30 \%$ 左右. 本文根据这一结果, 折算 出合金的平均摩尔质量 $M=92.1 \mathrm{~g} \cdot \mathrm{mol}^{-1}$, 平均密度 $\rho=11.08 \mathrm{~g} \cdot \mathrm{cm}^{-3}$. 由图 6 可知, 理论拟合曲线与实验 曲线较为吻合; 由表 1 可知, 通过非线性拟合得到不 
表 1 根据 HT 模型拟合得到的钯镍合金在不同阶跃电位下 的成核参数

Table 1 Kinetic parameters extracted from the current transients according to $\mathrm{HT}$ model

\begin{tabular}{cccc}
\hline Step potential $/ \mathrm{V}$ & $10^{5} \mathrm{D} /\left(\mathrm{cm}^{2} \cdot \mathrm{s}^{-1}\right)$ & $10^{-4} N_{0} / \mathrm{cm}^{-2}$ & $A / \mathrm{s}^{-1}$ \\
\hline-0.85 & 4.03 & 2.77 & 0.83 \\
-0.87 & 4.02 & 2.91 & 0.92 \\
-0.88 & 4.01 & 4.93 & 1.03 \\
-0.90 & 4.00 & 5.47 & 2.99 \\
-0.92 & 4.00 & 7.09 & 7.71 \\
\hline
\end{tabular}

$D$ : diffusion coefficient; $N_{0}$ : density of active nucleation sites; $A$ : nucleation rate constant

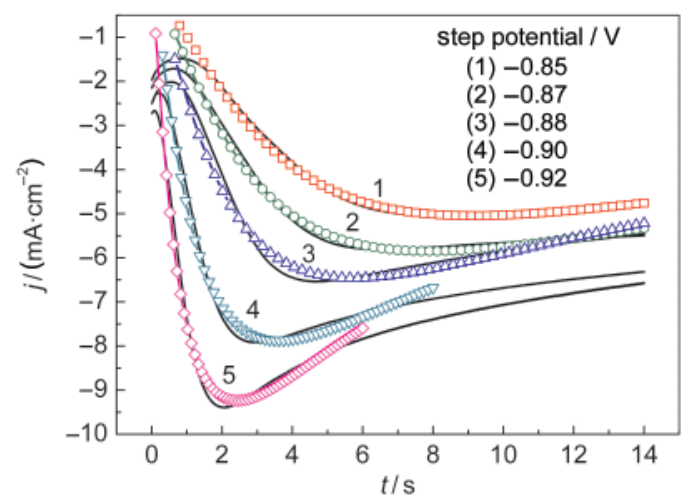

图 6 计时安培实验曲线(实线)与 HT 模型拟合曲线 (点划线)对比图

Fig.6 Comparison between the experimental current transient (solid line) and the corresponding theoretical current transients (dot line) generated by non-linear fitting of HT

同电位下钯镍络合离子的平均扩散系数基本不变, 为 $4.00 \times 10^{-5} \mathrm{~cm}^{2} \cdot \mathrm{s}^{-1}$; 成核密度数与基底的表面状态 以及沉积过电位密切相关. Michailova 等 ${ }^{27}$ 报道指 出, 金属电结晶过程的成核密度数范围为 $10^{4}-10^{10}$ $\mathrm{cm}^{-2}$. 本实验中, 随着阶跃电位从 $-0.85 \mathrm{~V}$ 负移 到 $-0.92 \mathrm{~V}$, 钯镍合金电结晶的成核密度数从 $2.77 \times$ $10^{4} \mathrm{~cm}^{-2}$ 增加到 $7.09 \times 10^{4} \mathrm{~cm}^{-2}$, 说明阶跃电位的负移 对电极表面起到活化作用, 使表面具有更多的活性 位点. 若析氢反应的变化及影响较小, 成核数密度 的增加则有利于生成晶体颗粒较细, 结构更加致 密、性能更好的钯镍合金镀层. 成核速率常数同样 随着该阶跃电位的负移从 $0.83 \mathrm{~s}^{-1}$ 提高到 $7.71 \mathrm{~s}^{-1}$.

\section{4 结 论}

以可工业化应用的钯镍合金电解液为研究体 系(浓度相对较高、合金体系更加复杂, 实验结果更 接近于实际), 采用循环伏安和恒电位计时安培曲线 方法, 成功地揭示了钯镍合金的电结晶机理并获得 相关的成核过程参数. 实验结果可为钯镍合金的电
沉积提供理论指导, 也为其他合金电结晶机理的研 究提供借鉴. 钯镍合金在玻碳电极上表现为扩散 控制下的不可逆电极反应过程, 并趋向于三维生 长的连续成核机理. 随着阶跃电位从 $-0.85 \mathrm{~V}$ 负移 到 $-0.92 \mathrm{~V}$, 钯镍合金电结晶的成核密度数增加, 成 核速率变大. 钯镍配合离子在此体系中的平均扩散 系数为 $4.00 \times 10^{-5} \mathrm{~cm}^{2} \cdot \mathrm{s}^{-1}$.

\section{References}

(1) Fleischmann, M.; Liler, M. Trans. Faraday Soc. 1958, 54, 1370.

(2) Fleischmann, M.; Thirsk, H. R. Trans. Faraday Soc. 1955, 51, 71.

(3) Fleischmann, M.; Thirsk, H. R. Electrochim. Acta 1958, 1, 146.

(4) Gunawardena, G. A.; Hills, G.; Montenegro, I.; Scharifker, B. J. Electroanal. Chem. 1982, 138, 225.

(5) Scharifker, B.; Hills, G. Electrochim. Acta 1983, $28,879$.

(6) Scharifker, B.; Mostany, J. J. Electroanal. Chem. 1984, 177, 13.

(7) Sluyters-Rehbach, M.; Wijenberg, J. H. O. J.; Bosco, E.; Sluyters, J. H. J. Electroanal. Chem. 1987, 236, 1.

(8) Heerman, L.; Tarallo, A. J. Electroanal. Chem. 1999, 470, 70.

(9) Hyde, M. E.; Compton, R. G. J. Electroanal. Chem. 2003, 549, 1.

(10) Franz, S.; Yasumura, K. Galvanotechnik 2002, 93, 1210.

(11) Hiramoto, O. ECS Transactions 2008, 3, 11.

(12) Abys, J. A.; Straschil, H. K.; Kadija, I. V.; Kudrak, E. J.; Blee, J. J. Metal Finish. 1991, 89, 43.

(13) Valcarcel, A.; Morfin, F.; Piccolo, L. J. Catal. 2009, 263, 315.

(14) Lou, Y.; Shao, Y. T.; Li, P.; Li, Z. L.; Niu, Z. J. J. Electroanal. Chem. 2008, 624, 33.

(15) Du, C. Y.; Chen, M.; Wang, W. G.; Yin, G. P.; Shi, P. F. Electrochem. Commun. 2010, 12, 843.

(16) Qiu, C. C.; Shang, R.; Xie, Y. F. ; Bu, Y. R. ; Li, C. Y.; Ma, H. Y. Mater. Chem. Phys. 2010, 120, 323.

(17) Shobha, T.; Aravinda, C. L.; Parthasarathi, B.; Gomathi, D. L.; Mayanna, S. M. Mater. Chem. Phys. 2003, 80, 656.

(18) Yang, F. Z.; Huang, L.; Xu, S. K.; Zhou, S. M. Acta Phys. -Chim. Sin. 2004, 20, 463. [杨防祖, 黄 令, 许书楷, 周绍民. 物 理化学学报, 2004, 20, 463.]

(19) Danaee, I.; Shoghi, F.; Dehghani, M. M.; Kameli, M. J. Solid State Electrochem. 2010, 14, 57.

(20) Safonova, T. Y. ; Khairullin, D. R.; Tsirlina, G. A.; Petrii, O. A.; Vassiliev, S. Y. Electrochim. Acta 2005, 50, 4752.

(21) Fletcher, S. Electrochim. Acta 1983, $28,917$.

(22) Fletcher, S.; Halliday, C. S.; Gates, D.; Westcott, M.; Lwin, T.; Nelson, G. J. Electroanal. Chem. 1983, 159, 267.

(23) Alvarez, A. E.; Salinas, D. R. Electrochim. Acta 2010, 55, 3714.

(24) Bard, A. J.; Faulkner, L. R. Electrochemical Methods: Fundamental and Applications, 2nd ed.; John Wiley: New York, 2001; pp 228-239.

(25) Feng, G.; Xiong, Y.; Wang, H.; Yang, Y. Electrochim. Acta 2008, $53,8253$.

(26) Heerman, L.; Tarallo, A. J. Electroanal. Chem. 1998, 451, 101.

(27) Michailova, E.; Milchev, A. J. Appl. Electrochem. 1991, 21, 170. 